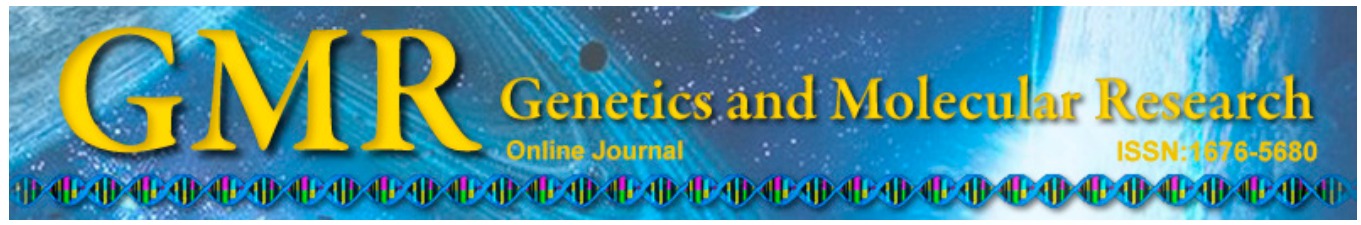

\title{
Genetic diversity analysis of tree peony germplasm using iPBS markers
}

\author{
Y.B. Duan ${ }^{1}$, D.L. Guo ${ }^{2}$, L.L. Guo ${ }^{1}$, D.F. Wei ${ }^{1}$ and X.G. Hou ${ }^{1}$ \\ ${ }^{1}$ College of Agriculture, Henan University of Science and Technology, \\ Luoyang, Henan Province, China \\ ${ }^{2}$ College of Forestry, Henan University of Science and Technology, \\ Luoyang, Henan Province, China \\ Corresponding author: X.G. Hou \\ E-mail: hxg382@126.com
}

Genet. Mol. Res. 14 (3): 7556-7566 (2015)

Received November 24, 2014

Accepted April 7, 2015

Published July 3, 2015

DOI http://dx.doi.org/10.4238/2015.July.3.31

\begin{abstract}
We examined the genetic diversity of 10 wild species (populations) and 55 varieties of tree peony using inter-primer binding site (iPBS) markers. From a total of 36 iPBS primers, 16 were selected based on polymorphic amplification. The number of bands amplified by each primer ranged from 9 to 19 , with an average of 12.88 bands per primer. The length of bands ranged from 100 to $2000 \mathrm{bp}$, concentrated at 200 to $1800 \mathrm{bp}$. Sixteen primers amplified 206 bands in total, of which 173 bands were polymorphic with a polymorphism ratio of $83.98 \%$. Each primer amplified 10.81 polymorphic bands on average. The data were then used to construct a phylogenetic tree using unweighted pair group method with arithmetic mean methods. Clustering analysis showed that the genetic relationships among the varieties were not only related to the genetic background or geographic origin, but also to the flowering phase, flower color, and flower type. Our data also indicated that iPBS markers were useful tools for classifying tree peony germplasms and for tree peony breeding, and the specific bands were helpful for molecular identification of tree peony varieties.
\end{abstract}

Key words: Genetic diversity; Germplasm resources; iPBS molecular markers; Paeonia suffruticosa Andrews 


\section{INTRODUCTION}

Tree peony (Paeonia L.) is a famous ornamental flower, frequently planted in gardens nearly worldwide. Tree peony is unique in China because cultivated varieties originated in China (Hong and Pan, 1998). Tree peony from other countries was directly or indirectly introduced from China (Zhao et al., 2004). Flowers of tree peony are large, colorful, and fragrant, known as the king of the flowers. There are currently more than 1000 varieties cultivated in China, which are divided into 9 cultivar groups according to the latest classification system of tree peony ( $\mathrm{Li}$ et al., 2011b). Most of these varieties are largely cultivated in the regions of Henan, Gansu, Shanxi, Sichuan, and Tibet, among others. With long-term domestication and cultivation as well as natural and artificial selection, tree peony possesses great genetic diversity. In addition, tree peony may be bred using many method, making the genetic relationships among species unclear and creating difficulties in distinguishing varieties and the breeding of new varieties (Zhou et al., 2003). Therefore, it is necessary for further analyze the diversity of the tree peony germplasm.

Molecular marker technology can detect the genetic diversity of an organism at the DNA level. This technology is not dependent on gene expression and environmental conditions, and the operation is simple, quick, and easily automated. Molecular markers play an important role in molecular marker-assisted breeding, population genetic studies, germplasm resource identification, genetic relationship analysis, and genetic map construction. Currently, genetic diversity studies of tree peony are mainly concentrated at the phenotypic level. The rapid development of molecular biology has provided a method for studying tree peony resources at the DNA level, including random-amplified polymorphic DNA (RAPD) (Hosoki et al., 1997; Chen et al., 2002; Meng and Zheng, 2004), simple-sequence repeats (Yuan et al., 2010; Hou et al., 2011; Gao et al., 2013), inter-simple sequence repeat (Li et al., 2011a), amplified fragment length polymorphism (Hou et al., 2006a), sequence-related amplified polymorphism (Han et al., 2008; Guo et al., 2009; Wang et al., 2011), and conserved DNA-derived polymorphism (Wang et al., 2014). However, different markers have different specificities, and studies have shown inconsistent results, thus, showing the need for additional methods. Retrotransposons are widely distributed in the genome of higher plants in high copy numbers (Hou et al., 2012). Among these, the long terminal repeat (LTR) retrotransposons are effective for plant gene function analysis and germplasm evaluation because of its unique nature (Guo et al., 2014b). Cloned LTR sequences are important in the theory and practice of studying plant diversity, genetic breeding, and species (Kalendar et al., 2011; Schulman et al., 2012; Guo et al., 2014c).

The inter-primer binding site (iPBS) is a new and effective method for separating LTR retrotransposon sequences using polymerase chain reaction (PCR) amplification of primer binding site intervals in LTR retrotransposons (Kalendar et al., 2010). This method differs from earlier retrotransposon-based markers because it is applicable not only to endogenous retroviruses, but also to both Gypsy and Copia LTR retrotransposons (Melnikova et al., 2012). Furthermore, the iPBS amplification technique is powerful for DNA fingerprinting technology without the need for prior sequence knowledge. Therefore, use of the iPBS marker is a simple and quick method that can be used to directly screen primers and study plants. This technology has been successfully applied in barley, wheat, apples, corn (Kalendar et al., 2010), flax (Smykal et al., 2011), apricot (Baranek et al., 2012), Saussurea (Gailite and Rungis, 2012), Cicer (Andeden et al., 2013), and guava (Asim et al., 2013). It is also suitable for studying the 
genetic diversity of germplasm resources and identifying grape species (Guo et al., 2014a). To provide a foundation for studies to explore, utilize, and preserve tree peony germplasm resources, we analyzed the genetic diversities of 10 wild species (populations) and 55 tree peony varieties using the iPBS technique.

\section{MATERIAL AND METHODS}

Ten wild species (populations) and 55 tree peony varieties were collected in April 2013. Table 1 summarizes the information of specimens used in this study. The 10 wild species were collected from the Gansu Forestry Extension Station of Science \& Technology, and the 55 tree peony varieties, including Zhongyuan Mudan, Xibei Mudan, Jiangnan Mudan, Japanese varieties, American varieties, and French varieties, were collected from the Luoyang National Peony Garden and Luoyang International Peony Garden. Genomic DNA was extracted from fresh, healthy leaves using a modified cetyltrimethylammonium bromide method as proposed by Guo et al. (2009). The DNA samples were stored at $-20^{\circ} \mathrm{C}$ until PCR amplification.

\begin{tabular}{|c|c|c|c|c|c|}
\hline Code & Cultivar & Place of origin & Code & Cultivar & Place of origin \\
\hline 1 & P. potaninii & Sichuan Yajiang & 34 & Huahongxiuqiu & Gansu \\
\hline 2 & P. lutea & Tibet Linzhi & 35 & Doulv & Luoyang \\
\hline 3 & P. jishanensis & Shanxi Jishan & 36 & Zihongchengyan & Luoyang \\
\hline 4 & Shengnongjia $P$. qiui & Hubei Shengnongjia & 37 & ShanhuTai & Luoyang \\
\hline 5 & P. rockii & Gansu Zhouqu & 38 & Yingluobaozhu & Luoyang \\
\hline 6 & P. ludlowii & Tibet Milin & 39 & Yinfenjinlin & Luoyang \\
\hline 7 & P. delavayi & Yunan Zhongdian & 40 & Shengdanlu & Luoyang \\
\hline 8 & P. decomposita & Sichuan Maerkang & 41 & Changhong & Anhui \\
\hline 9 & P. ostii & Henan Baotianman & 42 & Hongguanyuzhu & Gansu \\
\hline 10 & Heihaodao & America & 43 & Heidaogelasi & America \\
\hline 11 & Tianxiangzhanlu & Luoyang & 44 & Gejinzi & Luoyang \\
\hline 12 & Jinzhi & France & 45 & Wanhuasheng & Luoyang \\
\hline 13 & Jinhuang & France & 46 & Luhehong & Luoyang \\
\hline 14 & Huawang & Japan & 47 & Huhong & Anhui \\
\hline 15 & Bingshanfeicui & Gansu & 48 & Baiyu & Luoyang \\
\hline 16 & Yubanxiuqiu & Gansu & 49 & Yuloudiancui & Luoyang \\
\hline 17 & Yaohuang & Luoyang & 50 & Lvxiangqiu & Luoyang \\
\hline 18 & Jindao & America & 51 & Erqiao & Luoyang \\
\hline 19 & Huhong & Luoyang & 52 & Jiuzuiyangfei & Luoyang \\
\hline 20 & Luoyanghong & Luoyang & 53 & Qinglongwomochi & Luoyang \\
\hline 21 & Xishi & Anhui & 54 & Lantianyu & Luoyang \\
\hline 22 & Wulongpengsheng & Luoyang & 55 & Danluyan & Luoyang \\
\hline 23 & Hongguanyudai & Gansu & 56 & Huanghuakui & Luoyang \\
\hline 24 & Roufurong & Luoyang & 57 & Heihaijinlong & Luoyang \\
\hline 25 & Yinhongqiaodui & Luoyang & 58 & Aoyunshenghuo & Gansu \\
\hline 26 & Zhongguolong & America & 59 & Shouanhong & Luoyang \\
\hline 27 & Lanhaibibo & Luoyang & 60 & Mohaijinxue & Luoyang \\
\hline 28 & Xiaotaohong & Luoyang & 61 & Sanbiansaiyu & Luoyang \\
\hline 29 & Yinlinbizhu & Luoyang & 62 & Yingsuhong & Luoyang \\
\hline 30 & Yingrihong & Luoyang & 63 & Baokang P. qiui & Hubei Baokang \\
\hline 31 & Zipantuogui & Luoyang & 64 & Shibahao & Luoyang \\
\hline 32 & Zijinpan & Luoyang & 65 & Fomenjiasha & Luoyang \\
\hline 33 & Chunguihuawu & Luoyang & & & \\
\hline
\end{tabular}

Initially, 36 iPBS primers designed by Kalendar et al. (2010) were tested with 3 cultivars (LuoyangHong, Yaohuang, Erqiao) for PCR amplification. Primers generating strong and clearly amplified bands were selected. 
Conditions for iPBS-PCR amplification were conducted as described by Kalendar et al. (2010), with slight modifications. The $20-\mu \mathrm{L}$ reactions contained $1 \mathrm{X}$ PCR buffer, $0.3 \mu \mathrm{M}$ primers, $30 \mathrm{ng}$ DNA template, $0.4 \mathrm{mM}$ dNTPs, and $1.5 \mathrm{U}$ Taq DNA polymerase. PCR amplification was started with pre-denaturation at $95^{\circ} \mathrm{C}$ for $3 \mathrm{~min}$, followed by 30 cycles of denaturation at $95^{\circ} \mathrm{C}$ for $15 \mathrm{~s}$, annealing at $53^{\circ} \mathrm{C}$ for $60 \mathrm{~s}$, and extension at $68^{\circ} \mathrm{C}$ for $60 \mathrm{~s}$. Amplification was completed by holding the reaction mixture at $72^{\circ} \mathrm{C}$ for $5 \mathrm{~min}$. PCR-amplified products were detected on $1 \%$ agarose gels with $0.5 \mathrm{X}$ TBE (Trizma base, boric acid, EDTA) buffer at 5 $\mathrm{V} / \mathrm{cm}$ for $50 \mathrm{~min}$. Amplified products were stained with UltraPower ${ }^{\mathrm{TM}}$ before electrophoresis and visualized under UV light, and photographed under a white/ultraviolet transilluminator.

The amplified bands were scored as 1 for the presence and 0 for the absence of a band. Only clear, repeatable, and well-separated bands were selected for scoring. The number of polymorphic bands, the percentage of polymorphic bands, the effective number of alleles $\left(N_{\mathrm{E}}\right)$, Nei's gene diversity index $(H)$, and Shannon's information index $(I)$ were calculated using the POPGENE 32 software. The polymorphic information content was also calculated according to De Riek et al. (2001); polymorphic information content $=1-\left[f^{2}+(1-f)^{2}\right]$, where $f$ was the frequency of the marker. Pairwise comparisons between varieties were made using Nei and Li similarity coefficients with SIMQUAL module of NTSYS-pc (Rohlf, 2005). A dendrogram was constructed for all 65 varieties based on the unweighted pair group method with arithmetic mean using the SHAN module of NTSYS-pc. Principal coordinate analysis (PCoA) was performed to demonstrate the multiple dimensional distributions of the varieties in a scatterplot using NTSYS-pc.

\section{RESULTS}

Sixteen iPBS primers with clear, stable, and rich polymorphic amplification bands were selected from 36 primers designed by Kalendar et al. (2010) using Luoyanghong, Yaohuang, and Erqiao as DNA template materials (Table 2). A total of 206 bands were amplified in 65 tree peony accessions. The number of amplified bands produced using each primer varied from 9 to 19, with an average of 12.88. Primer 2255 amplified the fewest bands (9), while primer 2240 produced the largest number of bands (19). A total of 173 of the 206 bands amplified using 16 primers were polymorphic; the percentage of polymorphic bands ranged from $66.67 \%$ (2400) to $100 \%(2249,2253)$, with an average of $83.98 \%$ (Table 3$)$. Thus, iPBS markers were highly efficient when applied to detect the genetic diversity of tree peony. The results also showed that iPBS markers could be used to directly determine the genetic diversity of the tree peony germplasm. A representative fingerprinting pattern generated by primer 2373 is shown in Figure 1. The length of bands ranged from 100 to $2000 \mathrm{bp}$, concentrated from 200 to $1800 \mathrm{bp}$. There were significant differences between amplification products among tree peony varieties with the same primer, indicating that the tree peony cultivars had high polymorphism levels.

\begin{tabular}{|c|c|c|c|}
\hline Primer name & Sequence & Primer name & Sequence \\
\hline 2219 & 5'-GAACTTATGCCGATACCA-3' & 2253 & 5'-TCGAGGCTCTAGATACCA-3' \\
\hline 2221 & 5'-ACCTAGCTCACGATGCCA-3' & 2255 & 5'-GCGTGTGCTCTCATACCA-3' \\
\hline 2224 & 5'-ATCCTGGCAATGGAACCA-3' & 2256 & 5'-GACCTAGCTCTAATACCA-3' \\
\hline 2240 & 5'-AACCTGGCTCAGATGCCA-3' & 2373 & 5'-GAACTTGCTCCGATGCCA-3' \\
\hline 2241 & 5'-ACCTAGCTCATCATGCCA-3' & 2395 & 5'-TCCCCAGCGGAGTCGCCA-3' \\
\hline 2242 & 5'-GCCCCATGGTGGGCGCCA-3' & 2399 & 5'-AAACTGGCAACGGCGCCA-3' \\
\hline 2249 & 5'-AACCGACCTCTGATACCA-3' & 2400 & 5'-GAACTTGCTCCGATGCCA-3' \\
\hline 2252 & 5'-TCATGGCTCATGATACCA-3' & 2401 & 5'-AGTTAAGCTTTGATACCA-3' \\
\hline
\end{tabular}


Table 3. Results of the observed genetic diversity based on iPBS markers.

\begin{tabular}{lrrrrrr}
\hline Primer & TB & PB & PPB & PIC & DC & PDC \\
\hline 2219 & 14 & 10 & 71.42 & 0.378 & 46 & 70.77 \\
2221 & 12 & 10 & 83.33 & 0.358 & 40 & 61.54 \\
2224 & 12 & 9 & 75.00 & 0.340 & 44 & 67.69 \\
2240 & 19 & 14 & 73.68 & 0.298 & 44 & 67.69 \\
2241 & 10 & 8 & 80.00 & 0.335 & 27 & 41.54 \\
2242 & 13 & 12 & 92.31 & 0.392 & 38 & 58.46 \\
2249 & 13 & 13 & 100.00 & 0.431 & 54 & 83.08 \\
2252 & 14 & 12 & 85.71 & 0.360 & 58 & 89.23 \\
2253 & 12 & 12 & 100.00 & 0.334 & 46 & 70.77 \\
2255 & 9 & 7 & 77.78 & 0.413 & 39 & 60.00 \\
2256 & 14 & 13 & 92.86 & 0.324 & 39 & 60.00 \\
2373 & 13 & 12 & 92.31 & 0.297 & 44 & 67.69 \\
2395 & 14 & 11 & 78.57 & 0.369 & 52 & 80.00 \\
2399 & 10 & 9 & 90.00 & 0.314 & 32 & 49.23 \\
2400 & 15 & 10 & 66.67 & 0.299 & 40 & 61.54 \\
2401 & 12 & 11 & 91.67 & 0.418 & 32 & 49.23 \\
Average & 12.88 & 10.81 & 83.98 & 0.354 & 42.19 & 64.90 \\
\hline TB & tota & PB & & & &
\end{tabular}

$\mathrm{TB}=$ total bands; $\mathrm{PB}=$ polymorphic bands; $\mathrm{PPB}=$ percentage of polymorphic bands; $\mathrm{PIC}=$ polymorphic information content; $\mathrm{DC}=$ differentiable cultivars; $\mathrm{PDC}=$ percentage of differentiable cultivars.
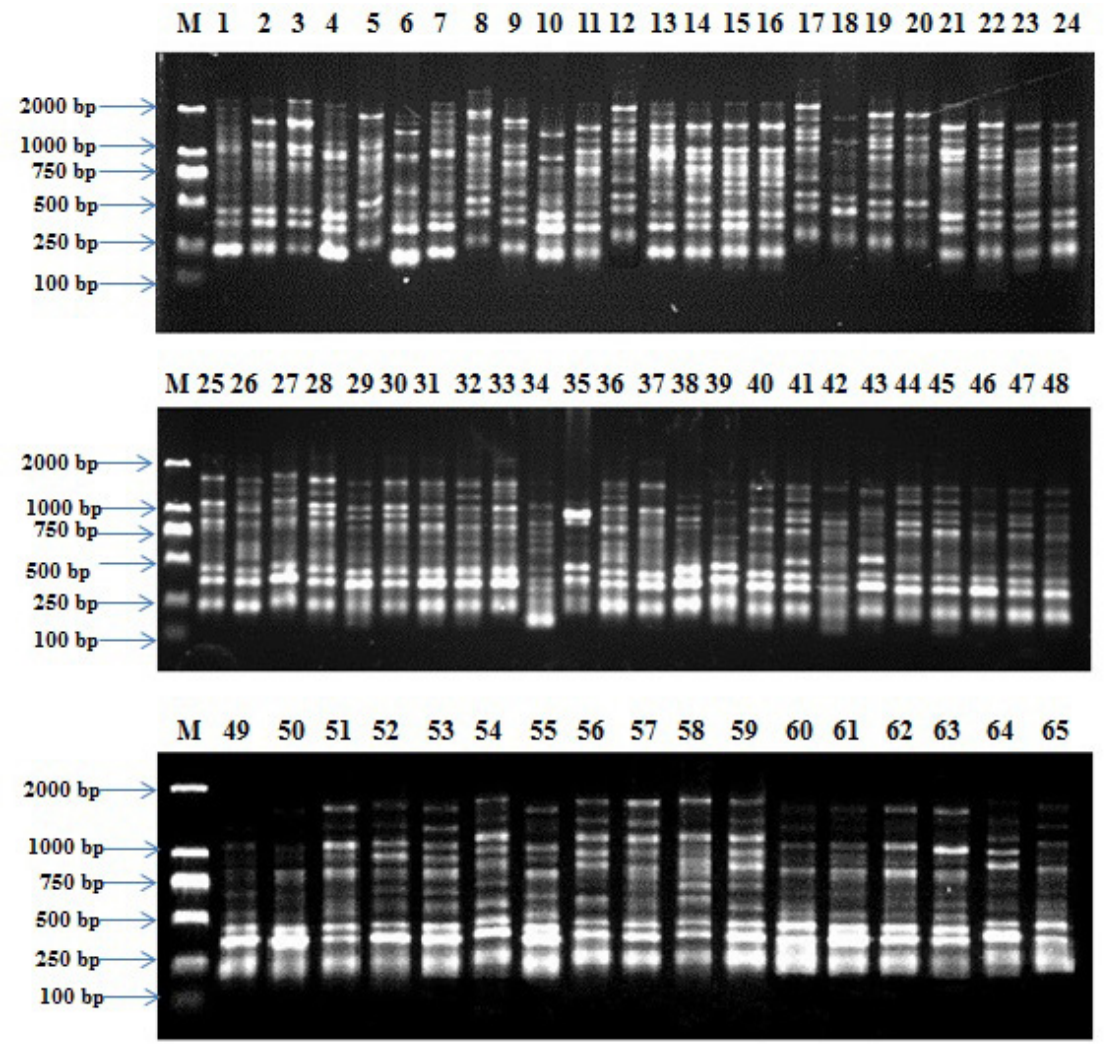

Figure 1. Agarose gel containing amplified products from the 2373 iPBS marker. The length of bands ranged from 100-2000 bp, concentrated from 200-1800 bp. The amplification products of each variety were different with the same primer. 
A total of 206 bands were used to calculate the genetic diversity among 65 tree peony accessions. Similarity coefficients among varieties were calculated using the NYSYS software. The results demonstrated that the similarity coefficient between the pairs of accessions varied from 0.46 to 0.99 among 65 tree peony varieties, mostly from 0.51 to 0.85 . The maximum coefficient was 0.99 between Yinhongqiaodui and Roufurong, indicating that the genetic distance between these varieties was the lowest and thus they had the closest relationship. In contrast, the relationship between Paeonia delavayi and Heihaidao was the most distant. The highest polymorphic information content value $(0.431)$ was observed for iPBS primer 2249 and the lowest (0.297) was for iPBS primer 2373 with an average of 0.354 , indicating that these loci were highly informative. In addition, $N_{\mathrm{E}}, H$, and $I$ were calculated using the POPGENE 32 software. The mean values of $N_{\mathrm{E}}, H$, and $I$ were $1.415,0.267$, and 0.421 , respectively. The genetic diversity degree among loci varied widely; for example, the highest values of $N_{\mathrm{E}}, H$, and $I$ were 2.000, 0.499, and 0.687 and the lowest were 1.016, 0.061, and 0.111 , respectively. All calculated indices confirm that there is abundant genetic diversity in tree peony.

The unweighted pair group method with arithmetic mean was used for cluster analysis and to construct a dendrogram. The clustering results showed that the genetic relationships between the tree peony germplasm were very complex. Figure 2 shows that 65 tree peony materials were clustered into 3 major clusters when 0.61 was chosen as the threshold. Cluster 1 included subsect Delavayanae for P. delavayi, Paeonia lutea, Paeonia potaninii, and Paeo-

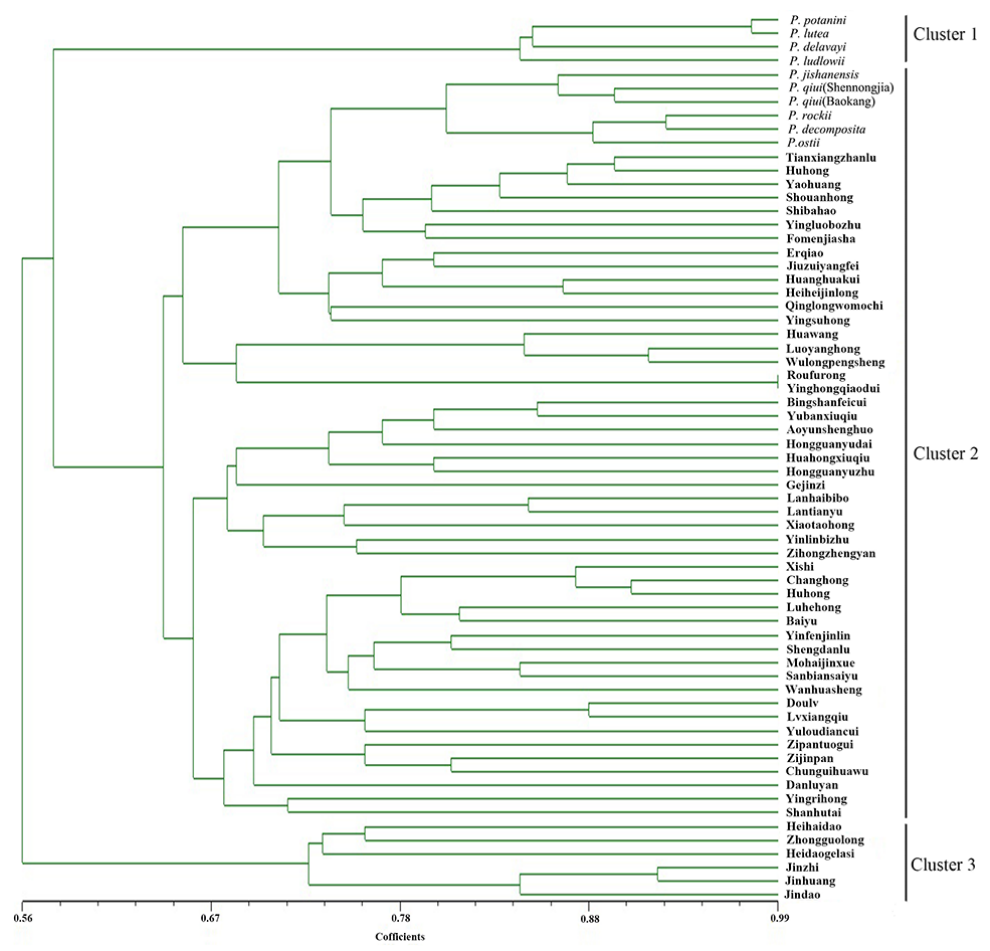

Figure 2. UPGMA dendrogram of 65 tree peony varieties based on 16 iPBS primers. 
nia ludlowii, and P. lutea and P. potaninii were the most closely related. Cluster 3 contained American varieties - Zhongguolong, Heihaidao, Heidaogelasi, and Jindao and French varieties - Jinhuang and Jinzhi. The remaining 55 peony samples belonged to Cluster 2.

Cluster 2 was clustered into 4 subgroups with a threshold of 0.67 . There were 6 wild species (populations) and 13 cultivated peonies in subgroup 1. The wild species (populations) Paeonia jishanensis, Shennongjia Paeonia qiui, and Baokang P. qiui clustered together, Paeonia rockii and Paeonia decomposita clustered together first, and then clustered with Paeonia ostii among the subgroup. These results indicated that the genetic relationship between $P$. jishanensis and $P$. qiui or $P$. rockii and $P$. decomposita was closest among the subsect Vaginatae. Additionally, the cultivars Tianxiangzhanlu, Huhong, Yaohuang, and Shouanhong clustered together because they all had crown-type flowers. Plants with red flowers, including Yingluobaozhu and Fomenjiasha, clustered together, and the individual plants were dwarfs. Huanghuakui clustered with Heihaijinlong because their flowers were similar to the lotus. Subgroup 2 included 5 cultivated peonies. In this study, Roufurong and Yinhongqiaodui clustered and their similarity coefficients were the highest possibly because they had the same flowering phase, flower type, and color. Luoyanghong was clustered with Wulongpengsheng, which may be related to the similar morphological features such as the purplish red flowers and rose-shaped flowers, among other features. There were 6 Northwest varieties Bingshanfeicui, Yubanxiuqiu, Aoyunshenghuo, Hongguanyudai, Huahongxiuqiu, and Hongguanyuzhu and 6 Central Plains' varieties Gejinzi, Lanhaibibo, Lantianyu, Xiaotaohong, Yinlinbizhu, and Zihongchengyan in subgroup 3. The 6 Northwest varieties were clustered to reflect the close geographic and genetic relationships. The crown-type flowers of Yinlinbizhu and Zihongchengyan clustered together. The blue flowers of Lanhaibibo and Lantianyu clustered together, while Gejinzi and Yinlinbizhu did not, although they all had purple flowers. Subgroup 4 contained 19 varieties. The Southern Yangtse varieties Xishi, Changhong, and Huhong were clustered in the subgroup. Among the other Central Plains varieties, the varieties with red flowers and the dwarf plant such as Yingrihong and Shanhutai clustered together. The genetic relationship between Donlv and Lvxiangqiu was close, as well as Shengdanlu and Yinfenjinlin, which all had pink flowers. However, varieties with white and crown-type flowers for Baiyu and Yuloudiancui were not clustered. Luhehong and Wanhuasheng had the same color and type flowers but were not clustered together.

The results showed a high correlation between genetic distance and geological origin by clustering of unweighted pair group method with arithmetic mean, and the tree peony varieties were clustered in different groups with geographic origin. These results are consistent with those of previous studies (Shi et al., 2012), while the flowering phase, flower color, and flower type of tree peony varieties had less impact on the classification of genetic groups (Chen et al., 2002; Hou et al., 2006a; Wang et al., 2011).

PCoA was performed to determine the associations between 65 tree peony specimens (Figure 3). In the diagram generated by PCoA, the foreign varieties separated from the others because they clearly had unique characteristics (Group 2) under the conditions of introduction country, and they can be used as new breeding resources. The specimens in the PCoA plot were identical to those in the dendrogram. The first 2 principal axes in the PCoA plot accounted for 11.98 and $8.66 \%$ of the total variations, respectively, indicating the complex multidimensional variation of the varieties. 


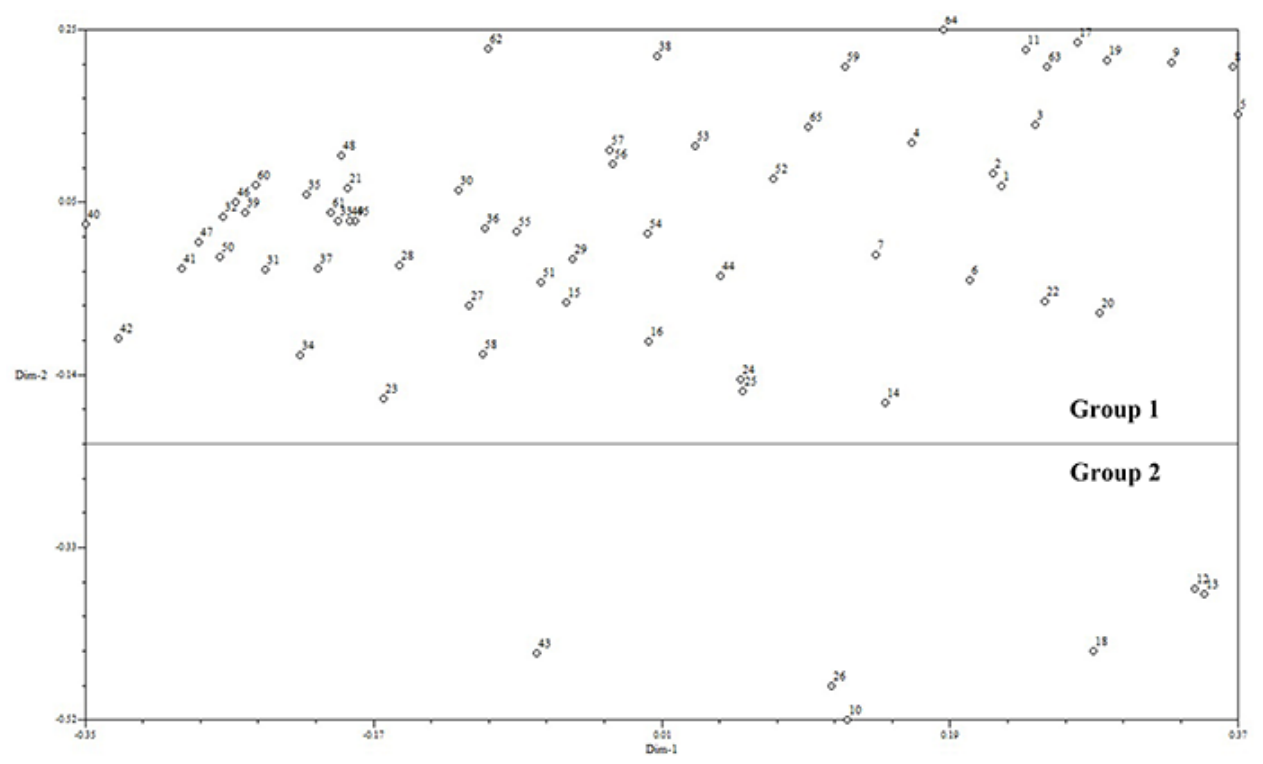

Figure 3. PCoA plot of the first 2 principal components of principal coordinate analysis based on iPBS data.

\section{DISCUSSION}

iPBS is a new molecular marker technology that does not require prior sequence information or the design of new primers, and it can be directly used for DNA fingerprinting technology and for the screening primers, greatly simplifying the experimental procedure. Furthermore, the results are highly reproducible because of the primer length and the high stringency achieved by the annealing temperature (Kalendar et al., 2010; Guo et al., 2014a). In this study, 36 iPBS primers were used to analyze 65 tree peony samples, and 16 primers with high polymorphism levels were screened. A total of 173 of 206 bands amplified were polymorphic, with a polymorphism ratio of $83.98 \%$, which was higher than that reported by Wang et al. (2011) (sequence-related amplified polymorphism, 69.6\%) in 20 tree peony cultivars with different flower forms, Chen et al. (2002) (RAPD, 80.6\%) in 35 tree peony cultivars derived from 7 different flower color types, and Meng and Zheng (2004) (RAPD, 80.1\%) in 23 tree peony specimens. The results indicated that the iPBS marker was a simple and efficient method according to its capacity to generate more polymorphic bands through PCR amplification. Although none of the iPBS primers could discriminate all the tree peony varieties, several primers distinguished all of the materials, including primers 2240, 2249, 2252, and 2395, demonstrating that iPBS can be used for classification and genetic diversity studies of tree peony specimens.

iPBS was used in tree peony varieties for genetic diversity analysis. Cluster analysis showed that the materials could be classified into 3 main clusters with several subclusters. Furthermore, clear discrimination between wild species (populations), particularly the subsect Delavayanae and cultivated varieties, were observed in the dendrogram (Figure 2), suggesting that genetic differences between these varieties were very high. The results showed that the relationship between $P$. jishanensis and P. qiui was closer, confirming these findings (Yuan 
and Wang, 1999; Zhao et al., 2004; Hou et al., 2006b), but it was inconsistent with the results found using RAPD or other methods (Zou et al., 1999; Meng and Zheng, 2004). Additionally, $P$. rockii and $P$. decomposita also showed a close relationship, which was consistent with the findings of previous studies (Zou et al., 1999; Tank and Sang, 2001; Meng and Zheng, 2004), However, Yuan and Wang (1999) did not reach the same conclusion.

In this study, 49 cultivars and subsect Vaginatae for P. jishanensis, P. qiui, P. rockii, $P$. decomposita, and $P$. ostii were in one group, which agreed with the findings of Meng and Zheng (2004). Yinhongqiaodui and Roufurong clustered and showed the highest similarity coefficients, possibly because they had the same flowering phase and chrysanthemum-flowered and pink flowers. In addition, they shared other similar morphological characteristics such as a purple halo at the base of petals, long leaves, and half-spreading. Yinhongqiaodui and Roufurong were bred in 1966 and 1975 in Heze Zhaolou, and the only difference among them was that there was tomentum in the back of leaves of Roufurong. Therefore, these varieties were difficult to distinguish ( $\mathrm{Li}$ et al., 2011b). Through the cluster analysis, we also found that species of the same origin were clustered together, indicating that geographic factors had a large impact on tree peony breeding. However, there were also some species clustering with different regions, particularly the Central Plains varieties. This may be related to the similar growing environmental conditions and artificial selection criteria. Additionally, it was the result of introduction between different regions or mutual penetration between certain genes of different species (Li et al., 2004). Species of the same origin were distributed into different groups, which may be because of mutation caused by long-term environmental induction and geographic changes (Wang et al., 2004). In this study, we also found that the flowering phase, flower color, and flower type of peony varieties impacted the definition of genetic groups, but did not show fully consistent relationships, which was consistent with the results of previous studies (Chen et al., 2002; Hou et al., 2006a; Wang et al., 2011). This also indicated that the origin of tree peony cultivated varieties were complex and diverse.

In summary, tree peony has accumulated a wealth of genetic diversity through longterm artificial introduction and natural selection. In this study, our results based on regional classification were better than those based on morphological clustering. Consistent with this observation, some tree peony varieties had a high molecular marker coefficient but numerous morphological differences. Therefore, in future studies, it is necessary to combine morphological characteristic and molecular markers to obtain more precise results, leading to more comprehensive and accurate analysis for genetic diversity and relationship determination of tree peony varieties.

In conclusion, this is the first study to use the iPBS method to study the genetic diversity of tree peony wild species (populations) and different cultivars. The results showed that iPBS is polymorphic and the tree peony germplasms have abundant genetic differences at the molecular level, providing a theoretical basis for tree peony resource evaluation and optimization and offer a new method for tree peony molecular markers as well.

\section{ACKNOWLEDGMENTS}

Research supported by the Natural Science Foundation of China (\#31370697), the Program for Science and Technology Innovation Talents in Universities of Henan Province (\#13HASTIT004), and the Support by Program for Innovative Research Team (in Science and Technology) in University of Henan Province (\#IRTSTHN014). 


\section{REFERENCES}

Andeden EE, Baloch FS, Derya M, Kilian B, et al. (2013). iPBS-Retrotransposons-based genetic diversity and relationship among wild annual Cicer species. J. Plant Biochem. Biotechnol. 22: 453-466.

Asim M, Muhammad JJ, Saeed A and Rashid A (2013). Evaluation of genetic diversity in open pollinated guava by iPBS primers. Pak. J. Agric. Sci. 50: 591-597.

Baranek M, Meszaros M, Sochorova J, Cechova J, et al. (2012). Utility of retrotransposon-derived marker systems for differentiation of presumed clones of the apricot cultivar Velkopavlovicka. Sci. Hortic. 143: 1-6.

Chen XM, Zheng GS and Li M (2002). RAPD-PCR analysis of genetic diversity of different color 35 tree peony cultivars. Sci. Agric. Sin. 35: 546-551.

De Riek J, Calsyn E, Everaert I, Van Bockstaele E, et al. (2001). AFLP based alternatives for the assessment of distinctness, uniformity and stability of sugar beet varieties. Theor. Appl. Genet. 103: 1254-1265.

Gailite A and Rungis D (2012). An initial investigation of the taxonomic status of Saussurea esthonica Baer ex Rupr. utilising DNA markers and sequencing. Plant Syst. Evol. 298: 913-919.

Gao ZM, Wu J, Liu ZA, Wang LS, et al. (2013). Rapid microsatellite development for tree peony and its implications. BMC Genomics 14: 886.

Guo DL, Hou XG and Zhang J (2009). Sequence-related amplified polymorphism analysis of tree peony (Paeonia suffruticosa Andrews) cultivars with different flower colours. J. Hortic. Sci. Biotechnol. 84: 131-136.

Guo DL, Guo MX, Hou XG and Zhang GH (2014a). Molecular diversity analysis of grape varieties based on iPBS markers. Biochem. Sys. Ecol. 52: 27-32.

Guo DL, Hou XG and Jia T (2014b). Reverse transcriptase domain sequences from tree peony (Paeonia suffruticosa) long terminal repeat retrotransposons: sequence characterization and phylogenetic analysis. Biotechnol. Biotec. Eq. 28 : 438-446.

Guo DL, Hou XG and Zhang X (2014c). A simple and efficient method to isolate LTR sequences of plant retrotransposon. Biomed Res. Int. 2014: 658473.

Han XY, Wang LS, Shu QY, Liu ZA, et al. (2008). Molecular characterization of tree peony germplasm using sequencerelated amplified polymorphism markers. Biochem. Genet. 46: 162-179.

Hong DY and Pan KY (1998). Taxonomical history and revision of Paeonia sect. Moutan (Paeoniaceae). Acta Phytotax. Sin. 37: 351-368.

Hosoki T, Kimura D, Hasegawa R, Nagasako T, et al. (1997). Comparative study of Chinese tree peony cultivars by random amplified polymorphic DNA (RAPD) analysis. Sci. Hortic. 70: 67-72.

Hou XG, Yin WL, Li JJ and Wang HF (2006a). AFLP analysis of genetic diversity of 30 tree peony (Paeonia suffruticosa Andr.) cultivars. Sci. Agric. Sin. 39: 1709-1715.

Hou XG, Yin WL, Li JJ and Wang HF (2006b). Phylogenetic relationship of dwarf tree peony cultivars by AFLP analysis. J. Beijing Forestr. Univ.-Chin. Edn. 28: 73.

Hou XG, Guo DL and Wang J (2011). Development and characterization of EST-SSR markers in Paeonia suffruticosa (Paeoniaceae). Am. J. Bot. 98: 303-305.

Hou XG, Zhang X and Guo DL (2012). Identification and analysis methods of plant LTR retrotransposon sequences. Hereditas 34: 1491-1500.

Kalendar R, Antonius K, Smýkal P and Schulman AH (2010). iPBS: a universal method for DNA fingerprinting and retrotransposon isolation. Theor. Appl. Genet. 121: 1419-1430.

Kalendar R, Flavell AJ, Ellis TH, Sjakste T, et al. (2011). Analysis of plant diversity with retrotransposon-based molecular markers. Heredity 106: 520-530.

Li BY, Zhou XM and Zhang QX (2011a). Establishment of core collection for tree peony cultivars from central China. Acta Agric. Boreal-Sin. 26: 100-105.

Li JJ, Zhang XF and Zhao XQ (2011b). Tree peony of China. Encyclopedia of China Publishing House, Beijing, 16-22.

Li XX, Zhu DW and Du YC (2004). Studies on genetic diversity and phylogenetic relationship of cucumber (Cucumis sativus L.) germplasm by AFLP technique. Acta Hortic. Sin. 31: 309-314.

Melnikova NV, Kudryavtseva AV, Speranskaya AS, Krinitsina AA, et al. (2012). The FaRE1 LTR-retrotransposon based SSAP markers reveal genetic polymorphism of strawberry (Fragaria x ananassa) cultivars. J. Agric. Sci. 4: 111.

Meng L and Zheng GS (2004). Phylogenetic relationship analysis among Chinese wild species and cultivars of Paeonia sect. Moutan using RAPD markers. Sci. Silvae Sin. 5: 17.

Rohlf F (2005). NTSYS-pc: Numerical taxonomy and multivariate analysis system, version 2.2. Exeter software, Setauket.

Schulman AH, Flavell AJ, Paux E and Ellis THN (2012). The application of LTR retrotransposons as molecular markers in plants. Methods Mol. Biol. 859: 115-153. 
Shi YT, Zhou B and Zhang XX (2012). Assessment of genetic diversity and relationship of 89 tree peony cultivars from different provenances. Acta Hortic. Sin. 39: 2499-2506.

Smykal P, Bacova-Kerteszova N, Kalendar R, Corander J, et al. (2011). Genetic diversity of cultivated flax (Linum usitatissimum L.) germplasm assessed by retrotransposon-based markers. Theor. Appl. Genet. 122: 1385-1397.

Tank DC and Sang T (2001). Phylogenetic utility of the glycerol-3-phosphate acyltransferase gene: evolution and implications in Paeonia (Paeoniaceae). Mol. Phylogenet. Evol. 19: 421-429.

Wang J, Guo DL, Hou XG, Fan BY, et al. (2011). SRAP analysis of genetic relationships of the different tree peony flower forms. Chin. Agric. Sci. Bull. 28: 032.

Wang XW, Fan HM, Li YY, Sun X, et al. (2014). Analysis of genetic relationships in tree peony of different colors using conserved DNA-derived polymorphism markers. Sci. Hortic. 175: 68-73.

Wang ZF, Sun RF, Sun XL, Gu XF, et al. (2004). Studies on genetic relationship among local Cucumber germplasm of Shandong province by AFLP analysis. Acta Hortic. Sin. 31: 103-105.

Yuan JH, Cheng FY and Zhou SL (2010). Hybrid origin of Paeonia x yananensis revealed by microsatellite markers, chloroplast gene sequences, and morphological characteristics. Int. J. Plant Sci. 171: 409-420.

Yuan T and Wang LY (1999). Pollen morphology of several tree peony wild species and discussion on its evolution and taxonomy. J. Beijing Forestr. Univ. 1: 17-21.

Zhao X, Zhou ZQ, Hong DY and Pan KY (2004). Molecular evidence for the interspecific relationships in Paeonia sect. Moutan: PCR-RFLP and sequence analysis of glycerol-3-phosphate acyltransferase (GPAT) gene. Acta Phytotax. Sin. 42: 236-244.

Zhou ZQ, Pan KY and Hong DY (2003). Advances in genetic relationship between wild group and cultivated species origin of Paeonia sect. Moutan (Paeoniaceae). Acta Phytotax. Sin. 30: 751-756.

Zou YP, Cai ML and Wang ZP (1999). Systematic studies on Paeonia sect. Moutan DC. based on RAPD analysis. Acta Phytotax. Sin. 37: 220-227. 